\title{
1 Novel shape indices for vector landscape pattern analysis
}

The formation of an anisotropic landscape is influenced by natural and/or human processes, which can then be inferred on the basis of geometric indices. In this study, two minimal bounding rectangles in consideration of the principles of mechanics (i.e. minimal width bounding (MWB) box and moment bounding (MB) box) were introduced. Based on these boxes, four novel shape indices, namely MBLW (the length-to-width ratio of MB box), PAMBA (area ratio between patch and MB box), PPMBP (perimeter ratio between patch and MB box) and ODI (orientation difference index between MB and MWB boxes), were introduced to capture multiple aspects of landscape features including patch elongation, patch compactness, patch roughness and patch symmetry. Landscape pattern was, thus, quantified by considering both patch directionality and patch shape simultaneously, which is especially suitable for anisotropic landscape analysis. The effectiveness of the new indices were tested with real landscape data consisting of three kinds of saline soil patches (i.e. the elongated shaped slightly saline soil class, the circular or half-moon shaped moderately saline soil, and the large and complex severely saline soil patches). The resulting classification was found to be more accurate and robust than that based on traditional shape complexity indices.

Keywords: landscape metrics; anisotropy; moment box; patch elongation; patch symmetry

\section{Introduction}

Landscape patterns may be defined as sets of landscape observations with spatial structure and which are, thus, significantly different from the realization of a random process. These patterns contain information on the mechanisms or processes from which they emerge (Grimm et al. 2005, Schröder and Seppelt 2006). Quantifying landscape patterns is, thus, considered to be a prerequisite for the study of patternprocess relationships (Turner 1990, Uuemaa et al. 2013), a fundamental pursuit of landscape ecology (Turner 2005, Helfenstein et al. 2014). Landscape pattern analysis based on the patch-matrix model (i.e. landscape pattern indices (LPIs)) or the gradient 
model (McGarigal et al. 2009) has, therefore, received increasing attention in both ecological research and the environmental management communities (Cissel et al. 1999, Fu and Chen 2000, Turner 2005).

In line with human interpretation of real landscapes (Lausch et al. 2015), landscape pattern indices (LPIs) offer an effective way to capture landscape structure, with either landscape-, class-, or patch-focus (McGarigal and McComb 1995, Kupfer 2012). This has increased our understanding of the relationships between spatial patterns and ecological processes on a range of scales (Wu 2013). As a popular quantitative analysis tool (Schröder and Seppelt 2006), LPIs have been applied increasingly to a variety of issues in landscape ecology (Uuemaa et al. 2013, Lausch et al. 2015), for example, assessment of landscape patterns or changes in land cover/use (Seto and Fragkias 2005; Reddy et al. 2013; Van Den Hoek et al. 2015), inference of landscape functions (Bolliger et al. 2007; Li et al. 2015), and quantification of ecosystem services (Syrbe and Walz 2012). The rapid advancement of remote sensing and geographic information systems (GIS) has also promoted the development and utilization of LPIs. During the past 30 years, numerous LPIs have been developed to quantify different spatial and compositional aspects of landscape structure (Lausch et al. 2015), and they are derived variously from fractal geometry (Krummel et al. 1987, Li 2000), information theory (O’Neill et al. 1988), percolation theory (Gardner and O’Neill 1991), statistical measures of dispersion (Gertsev 2004), mechanics (Zhang et 51 al. 2006) and mathematical morphology (Vogt et al. 2007). Most of these indices can be 52 computed readily by accessible software (e.g., 'r.le' and 'FRAGSTATS') to facilitate their implementation (Baker and Cai 1992, McGarigal and McComb 1995, Remmel and 54 Fortin 2013). 
In the face of complicated and diversified geographic landscapes, LPIs exhibit 56 certain deficiencies and limitations. In particular, some LPIs provide ambiguous 57 information about spatial patterns. For example, one landscape index may have the same numerical value for drastically different landscapes (Gustafson and Parker 1992, Tischendorf 2001, Corry and Nassauer 2005), while several visually distinct spatial 60 patterns may exhibit similar LPI values (Remmel and Csillag 2003, Turner 2005). One 61 important ambiguity is that most shape complexity indices (including many fractal methods) are derived based on a form of perimeter-area relationship (Forman and Godron 1986, Riitters et al. 1995, Gustafson 1998) and, for example, ignore the directional differences between patches. Current landscape metrics actually belong to indices of scalar quantity, that is, with loss of a patch's vector dimension (Zhang et al. 2006), which may result in uncertainties in shape identification. Considering a "curved" patch and an elongated linear patch, for example, both may have equal area and perimeter (i.e. their shape complexity or fractal indices might be exactly the same), but are nevertheless shaped distinctively.

Spatial anisotropy, the variation in spatial autocorrelation with orientation or direction, is often found in ecological variables because spatial patterns are sometimes produced by directional natural phenomena such as wind, fire, floods and tectonics (e.g. Legendre and Fortin 1989; Rossi et al. 1992; Gustafson 1998; Wu et al. 2000; Zhang et al. 2006). Meanwhile, human activities may also introduce a directional influence on landscapes. For example, tillage often leads to an anisotropic distribution of properties of the land surface (Vidal Vázquez et al. 2005). Moreover, spatial anisotropy is often associated with important ecological functions. For instance, landscape anisotropy has a direct effect on wetland flooding dynamics (Kaplan et al. 2012, Yuan et al. 2015) and the combined effects of soil anisotropy and topographic slope significantly affect the 
80 soil moisture regime by controlling the movement of water across and through the

81 landscape (Zaslavsky and Rogowski 1969). Spatial anisotropy, therefore, plays a crucial

82 role in real landscape analysis, which allows us to better understand the corresponding

83 landscape pattern-process relations and landscape functions. For example, based on

84 variogram and angular wavelet analysis, the directional process underpinning Bronze

85 Age surface pottery in the northern Murghab Delta was identified: specifically, the 86 impact of the complex system of watercourses in the delta on both settlement and post87 depositional processes (Markofsky and Bevan 2012). However, the variogram is a geostatistical tool and is, thus, not appropriate for quantifying anisotropy in terms of the 89 geometry of objects and, thus, related patch-based models. Consequently, it is necessary 90 to develop landscape indices by considering the shape properties of a patch and its 91 directional distribution simultaneously, that is, vector landscape pattern analysis (Zhang et al. 2006).

Zhang et al. (2006) first utilized the moment orientation (MO) index to represent patch orientation, based on planar characteristics defined by the principles of mechanics such as the moment of inertia, product of inertia and major/minor principal axes. The index was used to identify Qianan lakes (located in the central part of this paper's study area), whose orientations were heavily affected by the prevailing wind. However, shape complexity did not include the patch's anisotropy. Therefore, the minimum width bounding (MWB) box and the moment bounding (MB) box on the basis of the MO,

100 were introduced here simultaneously. Based on these two boxes, novel landscape 101 indices for vector landscape pattern analysis were proposed:

102 (1) patch length-to-width ratio,

103 (2) area ratio between patch and MB box,

104 (3) perimeter ratio between patch and MB box, 
107 different types of saline soils in the western part of the Songnen plain, China. These

108 different types of saline soil are located in different parts of a large paleolake that have

109 specific geographic conditions. Accurate discrimination of these saline soils would be

110 potentially useful for landscape management. However, while they vary from each other

111 in salinity level, they have similar remote sensing spectra. For this reason, classification

112 of the soil types based on traditional remote sensing classification approaches that

113 depend primarily on reflectance spectra is of limited accuracy. Consequently, we

114 investigate the additional class separability that can be attained by application of the

115 novel shape descriptors above to the landscape patches. While it is clear that anisotropy

116 plays a key role in determining landscape processes, or indicating the nature of the

117 underlying landscape processes, this paper seeks to test the specific hypothesis that

118 anisotropy and related shape indices can increase the accuracy of classification of

119 objects in the object-based image analysis (OBIA) sense. Since these indices can be

120 generated automatically, if they are ignored in classification analysis, this simply means

121 that the accuracy of classification may be less than it would be if they were included.

\section{Novel shape indices}

\section{2.1 Minimum Width Bounding (MWB) box}

124 The minimum width bounding (MWB) box, in computational geometry, generally

125 refers to the smallest enclosing rectangle with the least width over two-dimensional

126 space (Chaudhuri and Samal 2007). The properties of a MWB box are translation,

127 rotation and reflection invariance in terms of its enclosing polygon, thus, indicating the

128 corresponding orientation of the original polygon. 
130 spatial distribution of the vertices along the boundary of the polygon. A least square

131 linear regression is first applied to fit a line, followed by an axis transformation to the

132 local coordinate system. The bounding box can then be built up based on the maximum

133 projections of each vertex on the new axis. Since the vertex density and spatial

134 distribution often influence the size of the bounding box, which is not the desired MWB

135 box in most cases, the MWB box is searched numerically by the so-called "rotation

136 calliper" method given a user-defined threshold (Toussaint 1983). Detailed steps for

137 building the MWB box are given below:

$138 \quad$ Step 1: Least square approximation to fit a line (Stigler 1981)

139 The linear function minimizing the squared errors can be calculated as:

$$
f(x)=b_{0}+b_{1} x
$$

$$
b_{1}=\frac{\sum x y-n \overline{x y}}{\sum x^{2}-n(\bar{x})^{2}}
$$

$$
b_{0}=\bar{y}-b_{1} \bar{x}
$$

Where

$$
\bar{x}=\frac{1}{n} \sum_{i=1}^{n} x_{i}
$$

$$
\bar{y}=\frac{1}{n} \sum_{i=1}^{n} y_{i}
$$

$$
\sum x y=\sum_{i=1}^{n} x_{i} y_{i}
$$


In Equations 1 to 7 , the parameter $b_{1}$ is the slope of the fitted line, and the

150 variable $n$ is the number of vertices of each polygon.

Step 2: Coordinate transformation based on the estimated slope Coordinate transformation based on the fitted line is given by

$$
\theta=\arctan \left(b_{1}\right)
$$

Therefore, $\sin \theta$ and $\cos \theta$ for coordinate transformation can be calculated via Equation 8. Given a vertex $(x, y)$ in a global coordinate system with origin $\left(x_{0}, y_{0}\right)$, the new coordinate $\left(x^{\prime}, y^{\prime}\right)$ can be extracted by coordinate translation and rotation (Equation 9).

$$
\left(\begin{array}{c}
x^{\prime} \\
y^{\prime}
\end{array}\right)=\left(\begin{array}{cc}
\cos \theta & \sin \theta \\
-\sin \theta & \cos \theta
\end{array}\right)\left(\begin{array}{l}
x-x_{0} \\
y-y_{0}
\end{array}\right)+\left(\begin{array}{c}
b_{0} \cos \theta \\
b_{0} \sin \theta
\end{array}\right)
$$

By translating and rotating the axes, the $x$-axis in the new coordinate system is defined along the fitted line. A point on the $x$-axis is selected randomly as the origin of the new coordinate system, and the $y$-axis is defined perpendicular to the new $x$-axis.

Step 3: Finding the maximum and minimum coordinates of the vertices Under the new coordinate system, the maximum and minimum $y$-coordinates of the vertices, $Y_{\min }$ and $Y_{\max }$, as well as those of the $x$-coordinates, $X_{\min }$ and $X_{\max }$, can be determined, which then can be used as the initial minimum bounding box.

$$
\text { Step 4: Rotating calliper to search the MWB box numerically }
$$

The main axis fitted by least squares approximation is influenced largely by vertex density and distribution. Therefore, it is necessary to turn the initial minimum bounding box in discrete angular steps (Lewis et al. 1997) to locate the rectangle 
170 bounding box with minimum width, (i.e. the MWB box). The initial angle for each

171 rotation is set as $\theta$, iteratively increasing or decreasing by a small angle (predefined

172 as $\delta$ ) to find the bounding box with minimum width or approximate to the minimum,

173 which is the minimum width bounding (MWB) box with orientation $\theta_{M W B}$.

\section{$174 \quad 2.2$ Moment Bounding (MB) box}

175 The MB box is the minimal bounding rectangle built upon the moment orientation 176 (MO) (the orientation of the major axis), which is derived from planar characteristics 177 defined by mechanics (Zhang et al. 2006). The MO is reviewed briefly as follows:

178 Suppose that $(x, y)$ is a point within a planar polygon $(S)$ (Figure 1), whose

179 centroid is $C(\bar{x}, \bar{y})$, and the moment of inertia about the $x$-axis $\left(I_{x x}\right)$ and about the $y$ 180 axis $\left(I_{y y}\right)$, as well as the product of inertia $\left(I_{x y}\right)$, respectively, are expressed by 181 Equations 10, 11 and 12.

$$
I_{x x}=\int y^{2} d A
$$

$$
I_{x y}=\int x^{2} d A
$$

$$
I_{x y}=\int x y d A
$$
and Gere 1972). 
191 orientation of the major axis) is calculated by Equations 13 and 14 (Timoshenko and

192 Gere 1972). The moment bounding (MB) box that minimally encloses the polygon is

193 then constructed by taking $\theta_{M B}$ as the orientation of the long side of the MB box.

194 Equations 10-14, in discrete form suitable for patch computation, are deduced by 195 applying Green's theorem which relates the value of a line integral to that of a double 196 integral (see Zhang et al. (2006) for details).

$$
\tan 2 \theta_{M B}=\frac{2 I_{x y}}{I_{y y}-I_{x x}}
$$

\subsection{Novel shape indices}

200 Figure 2 shows the relations among a polygon (in black), its MWB box (in blue) and

201 MB box (in red). Here, $C$ is the centroid of the polygon. $P Q$ is the minor axis of the MB

202 box, about which the moment of inertia of the polygon is the maximum; $M N$ is the major axis of the MB box, about which the moment of inertia of the polygon is the minimum. $A B(E F)$, along the truck line of the long (short) side of the MWB box, is the major (minor) axis of the MWB box; $E^{\prime} F^{\prime}$ is the line passing through $C$ and parallel to the MWB box's long side. $\angle M C E$ ' is the angle between the two boxes, that is, the orientation difference between the two major axes $(M N$ and $E F)$ of the boxes. In the figure, $M N$ is deflected clockwise relative to $E F$, which indicates that the polygon is asymmetrically distributed between the two sides of $M N$, the major axis of the MB box.

210 The area of the polygon in the lower left quarter is much larger than the opposite.

Suppose the area and perimeter of a polygon are given by PA and PP, respectively; the area and perimeter of the MB box is MBA and MBP, respectively, the 
213 length and width of the MB box are L and W, respectively, and the orientation of the

214 MB box and MWB box are $\theta_{M B}$ and $\theta_{M W B}$, respectively.

$215 \quad$ Figure 2 is here.

216 If $\theta_{M B}=\theta_{M W B}$ (or $\left|\theta_{M B}-\theta_{M W B}\right|<\delta, \delta$ is a user-defined threshold), the patch is

217 symmetrical either around the major or the minor axis of the MB box. If symmetrical

218 around the minor axis of the MB box, the centroid of the polygon lies on the minor axis

219 of the MWB box (Figure 3(a)); if symmetrical around the major axis of the MB box, the

220 centroid of the polygon lies on the minor axis of the MWB box (Figure 3(b)). In either

221 situation, the centroid passes through the major and minor axes of the MB box

222 simultaneously.

$223 \quad$ Figure 3 is here.

224 Novel shape indices can then be derived (Table 1), including the MBLW (the 225 length-to-width ratio of MB box), PAMBA (area ratio between patch and MB box), 226 PPMBP (perimeter ratio between patch and MB box) and ODI (orientation difference 227 index between MB and MWB boxes).

Table 1 is here.

\section{Study area and data}

230 The study area is located between $122^{\circ} 03^{\prime} 41^{\prime \prime} \mathrm{E}-124^{\circ} 38^{\prime} 45^{\prime \prime} \mathrm{E}$ and $43^{\circ} 54^{\prime} 58^{\prime \prime} \mathrm{N}-$ $23145^{\circ} 45^{\prime} 50^{\prime}$ 'N, the hinterland of western Songnen Plain, Northeast China, covering the

232 western Jilin Province and the Inner Mongolia Autonomous Region (Figure 4). The 233 climate of this area is characterized as temperate continental monsoon ranging from 234 semi-humid to semi-arid with an annual average temperature of $4{ }^{\circ} \mathrm{C}$ (Chi and Wang 235 2010). Annual mean precipitation is around $370-400 \mathrm{~mm}$ with $80 \%$ of the rainfall in 236 July and August, causing a moisture deficit during 7 months of the year (Wang et al. 
2009). However, the annual evaporation reaches $1700-1900 \mathrm{~mm}$ on average, about 4-5

238 times greater than precipitation. Such high levels of evaporation result in large areas of 239 land degradation into saline soils throughout the study area. Figure 4 is here.

The salt-affected soils are developed by several natural environmental factors, such as climate, geology, parent material, hydrological conditions, and freeze-thaw.

243 There is evidence that a large paleolake in this area was formed after the Triassic Era by seawater incursion events due to tectonic activities (Huang et al. 2013). The paleolake gradually shrank in the Late Pleistocene due to the slow rise of the Songnen Plain and a long-term dry cold climate, and broke into hundreds of lake groups. These geological and geomorphological processes resulted in different degrees of salinity in different regions with distinctive geometric patterns. According to reference maps provided by

249 local experts and soil scientists, the saline soils comprise of slightly saline, moderately saline, severely saline and "other" classes. The slightly saline soils along the large paleolake shore, are geographically located at the southern shore of the large paleolake with strongly oriented and elongated patterns; the moderately saline soils are distributed around current lakes with circular or half-moon shapes; the severely saline soils mostly lie in the central region of the large paleolake, which are large sized, irregularly distributed over the space with some connections between them (Qiu et al. 2012); the "other" saline soil type is uncertain in geometry, location and saline degree and, thereby, is ignored in this study.

Three cloud-free scenes acquired by the Landsat 8 OLI sensor on 15 September 2592014 (Path 120, Row 28-29 and Path 119 Row 29) were used in this research. The 260 images were composed of seven multispectral bands (Coastal Aerosol, Blue, Green, 261 Red, NIR, SWIR1 and SWIR2) with a spatial resolution of $30 \mathrm{~m}$. After radiometric and 
262 geometric correction, the images were segmented by a multi-resolution segmentation

263 algorithm followed by spectral difference segmentation using the eCognition software

264 to obtain vector or polygon data representing the saline soil patches with an overall

265 classification accuracy of $90 \%$. These saline soil vector polygons form the input data for

266 the landscape pattern analysis and for validating the method. Note, because of the high

267 spectral similarity, different saline soil type patches are unable to be discriminated 268 based on spectra alone.

269 Ancillary data used in this paper, mainly as reference, include: 1) the National

270 Land Cover Database (NLCD) of China to check the segmentation results, 2) Reference

271 maps of different saline soil types provided by local experts for classification validation,

272 3) Obview-3 Panchromatic images and other fine spatial resolution imagery for visual

273 interpretation, and 4) geophysical data (ASTER GDEM and Geomorphological Map) of

274 the study area to understand the potential driving forces of landscape pattern. All these

275 data were pre-processed and stored in ArcGIS coverage within the same coordinate 276 system.

\section{$277 \quad 4$ Results}

\subsection{Saline soil feature extraction based on rules involving novel shape indices}

279 The feature extraction rules for each saline soil type were built on novel shape indices, 280 in which the thresholds for each parameter were established using a mix of expert 281 opinion (from saline soil scientists) coupled with a small amount of trial and error. The

282 final rule sets for feature extraction for the three saline soil classes, namely the slightly 283 saline soil, moderately saline soil and severely saline soil, are listed in Table 2, which 284 will be elaborated as follows:

Table 2 is inserted here. 
The slightly saline soil patches are located mainly in the southern shore of the

287 large paleolake. They are characterized by strong patch symmetry around the major axis

288 of the MB box and patch elongation with roughly east-west orientation, resulting in a

289 very small threshold of ODI (<=4.6) and a large threshold of MBLW (> 3); in addition,

290 the slightly saline soil patches have a relatively larger PAMBA (>0.34). Figure 5(a)

291 illustrates a region of such saline soil patches, each of which has a narrow, long and

292 almost coincident MWB box (in blue) and MB box (in red).

293 Surrounding current lakes, the moderately saline soil patches are usually

294 characterized as having circular or half-moon shapes, that is, the patches are curved

295 rather than elongated. Therefore, they have a low MWBLW $(<2.8)$ and a low PAMBA

296 value, within $(0.18,0.57)$; at the same time, they have a low PPMBP $(<2.22)$ in

297 comparison with severely saline soil. Figure 5(b) demonstrates a region of such saline

298 soil patches together with their MWB and MB boxes. From the figure, it can be seen

299 that the PAMBA and the PPMBP of the patches are small, and the MBLW is also

300 relatively small, with some MB boxes even close to square. Additionally, unlike the

301 slightly saline soil patches, the MWB and MB boxes of some moderately saline soil

302 patches are clearly not coincident (i.e. having relatively large ODI values).

303 Patches of severely saline soil are usually distributed at the centre of the large

304 paleolake, commonly with contagion between them, with large shape size and a high

305 shape complexity. The feature extraction rules for the severely saline soil patches were

306 developed using a large threshold $(>4,000.00 \mathrm{ha})$ of patch area and a large value of

307 PPMBP (> 3.4). The resulting features, thus, have large areas with geometrically 308 irregular shapes, as illustrated by Figure 5(c).

$309 \quad$ Figure 5 is inserted here. 
311 soil type (Figure 6) was produced, which includes four kinds of saline soils (i.e. slightly

312 saline soil, moderately saline soil, severely saline soil and other saline soils). It should

313 be noted that, the other saline soils were not identified with feature extraction rules;

314 instead, they were identified as the residual patches not identified as one of the three

315 former kinds. As the figure shows, the slightly saline soil consists of 45 patches (in

316 green), distributed mainly in the south, coinciding with the southern shore of the large

317 paleolake; the moderately saline soil class is composed of 127 patches (in blue),

318 distributed mainly in the east, a place where current lakes are widespread and occupied

319 by the interior of the large paleolake; the severely saline soil type includes five large

320 and highly contagious patches (in reddish orange), located mainly in the north,

321 coinciding with the centre of the large paleolake. The patch numbers, the mean patch

322 size, total area, mean patch perimeter and total patch perimeter of each saline soil class

323 were computed and are listed in Table 3. The saline soil classification accuracy was

324 further assessed using stratified random sample points collected from reference maps

325 provided by experts in paleogeography and soil science. The overall accuracy of the

326 saline soil classification is up to $92.23 \%$ with a Kappa index of 0.84 , which is a highly

327 accurate classification result.

$328 \quad$ Figure 6 is inserted here.

$329 \quad$ Table 3 is inserted here.

$330 \quad 4.2$ Feature separability of novel and traditional shape indices

331 The transformed divergence (TD) separability and Jeffries-Matusita (JM) distance

332 (italic) statistics for the novel indices, to be used in defining the rule sets for classifying

333 the three saline soil classes, are summarized in Table 4. Here, the values in bold font

334 indicate the high separability of a specific saline soil type from other classes based on 
335 the corresponding rule sets. In general, high separability (mostly greater than 1.8) was

336 achieved by the proposed shape indices used to define the rule sets for each saline soil

337 class. In terms of the slightly saline soil class, the three indices, namely ODI, MBLW

338 and PAMBA, obtained a very high TD separability, larger than 1.9 , even up to 2

339 (perfectly separable) when differentiating from the severely saline soil classes.

340 Meanwhile, low TD and JM $(1.4843,1.3408)$ between moderately saline soil and

341 severely saline soil were realized for the three indices, but this has no impact on the

342 feature extraction of the saline soil class in question (i.e. the slightly saline soil). With

343 respect to the moderately saline soil class, the three novel indices (i.e. MBLW, PAMBA

344 and PPMBP), also produced a very high TD separability (>1.9), and a high separability

345 (around 1.8) is, surprisingly, produced between the two other saline soil classes (the

346 slightly saline soil and the severely saline soil). As for the severely saline soil class, a

347 perfect separability (around 2) was realized by patch area and PPMBP. But a very low

348 TD and JM $(1.3408,0.8335)$ between the slightly saline soil and the moderately saline

349 soil occurred in this circumstance, revealing the inability of these two indices to

350 distinguish the two saline soil classes.

$351 \quad$ Table 4 is inserted here.

As benchmarks, three traditional shape indices including the perimeter-area ratio (PARA) (Baker and Cai 1992, Hulshoff 1995, Garrabou et al. 1998, Saura and Carballal 2004), fractal dimension (FRAC) (Feder 1988, Leduc et al. 1994), and shape index (SI)

355 (Saura and Carballal 2004) (see Table 5 for their detailed description) were tested for 356 discriminating jointly between the three saline soil classes. The corresponding TD 357 separability and JM (italic) values were computed and listed in Table 6. It can be seen from the Table 6 that, using the traditional indices, only the slightly and the severely saline soil classes are separable with high TD separability (>1.8), while the separability 
360 (1.2962) between the slightly and the moderately saline soil classes and that (1.617)

361 between the severely and the moderately saline soil classes are all relatively low.

Table 5 is inserted here.

Table 6 is inserted here.

\section{Discussion}

365

366

367

368

369

370

371

372

373

374

375

376

377

378

379

380

381

382

383

\subsection{Minimum bounding rectangles}

The minimum area bounding (MAB) box (i.e. the region bounding rectangle enclosing the minimum area) and its corresponding length-to-width ratio has been used to characterize the elongatedness of image objects, mainly for the purpose of remote sensing classification (Lewis et al. 1997, Jiao et al. 2012). However, when emphasising the minimum area of a rectangle, the patch directionality deriving from the ratio between the length and width of the rectangle is commonly ignored. The MB box, however, is built upon the moment orientation (MO), in which both the position and the area distribution of the patch (i.e. the inner structure of the patch) are taken into account (Zhang et al. 2006). Thus, it is a sensitive way to represent patch orientation. As shown by Figure 7 , the directional deviation of the patch between the MAB box and the MB box is the greatest. As for the MWB box, due to the consideration of the minimum width of the rectangle, its length is highlighted, thereby enhancing its capability to represent patch directionality. As exemplified by Figure 7, the MWB box lies in the middle of the MAB box and the MB box, but closer to the MB box. From the mechanical point of view, the MB box is exactly constructed by two orthogonal principal stresses along the major axis and the minor axis, respectively (Timoshenko and Gere 1972). Such a mechanical characteristic is basically captured with the MWB box, except that the MWB box is invariant as long as the change of patch area and 
384 distribution remains within the current MWB box. Thus, while the MB box acts as a 385 sensitive "detector" of patch geometry, the MWB box can serve as a benchmark. In fact, 386 the formation of an anisotropic landscape can be regarded as the influence of natural

387 and/or human forces, which can then be explained on the basis of mechanics. For 388 anisotropic (i.e. vector) landscape analysis, therefore, the introduction and adoption of 389 the MB and MWB boxes (both in possess of mechanical characteristics), instead of the 390 MAB box, would be theoretically sound, despite the small (or even no) differences 391 between them in some cases. Figure 7 inserts here.

\subsection{Novel shape indices}

394 Four novel shape indices, namely patch length-to-width ratio (MBLW), area ratio between patch and MB box (PAMBA), perimeter ratio between patch and MB box (PPMBP) and orientation difference between MB and MWB boxes (ODI), were derived on the basis of the two different bounding boxes (i.e. MB and MWB boxes). Multiple aspects of patch-based landscape information including patch elongation, patch compactness, patch roughness and patch symmetry can, thus, be captured, which are especially needed for anisotropy-based landscape analysis. The effectiveness of the proposed indices were tested with real landscape data consisting of the three saline soil classes, namely slightly saline soil, moderately saline soil, and severely saline soil. These self-patterned patches of different saline soil classes are located in different

404 geological and geographical environments (along the shore of the large paleolake, 405 surrounding current lakes, lying in the centre of the large paleolake); they were developed under distinctive geophysical processes and formed with different landscape

407 patterns including strip-like (elongated) shapes, circular or half-moon shapes, and large 408 and irregular shapes (Qiu et al. 2012). The proposed indices were able to capture 
multiple aspects of patch-based landscape information relating to each saline soil class,

410 with high TD separability values achieved for all pairs of saline soil classes (Table 4),

411 even up to a very high separability between the slightly saline soil class and other

412 classes. Traditional shape indices derived based on perimeter-area relationships (Saura 413 and Carballal 2004), in contrast, attained low TD values for all pairs of saline soil 414 classes except for the moderately saline soil and the severely saline soil (Table 6). These

415 indices had difficulty in distinguishing some anisotropic and non-anisotropic patches,

416 due to the existence of similar or even equal perimeter-area values among them.

\section{$417 \quad 5.3$ General applicability of novel shape indices}

418 The new boxes and indices proposed in this paper support quantitative modelling and

419 analysis of anisotropic landscapes. Moreover, the formation of anisotropic landscapes is 420 often associated with natural and/or anthropogenic driving forces. Each of the proposed 421 indices captures a particular ecological characteristic, which can aid ecological 422 interpretation and understanding. For example:

423 (i) the patch length-to-width ratio (MBLW) reflects the degree of anisotropy;

(ii) the area ratio between a patch and its MB box (PAMBA) indicates whether

(iii) the perimeter ratio between a patch and its MB box (PPMBP) reflects the roughness of an anisotropic patch, which is a measure of the natural degree of the patch boundary. It can also be used to quantify the contagiousness of a 
landscape patch, for instance, the high PPMBP value of the severely saline soil patches explains the obvious contagiousness of the patches. relationship of various anisotropic landscape patterns can be better understood,

437 primarily in landscape ecology, but also in a wide range of other potential fields (e.g. 438 sand dune development, forest fire spread, flood modelling, etc.).

\subsection{Limitations of the novel indices}

440 Novel indices were proposed for anisotropic, vector-based landscape analysis. For those

441 patches whose length-to-width ratio is close to 1 , application of these indices can lead to

442 some uncertainties. Further, the new indices might be less sensitive to shape complexity

443 for non-anisotropic landscape patterns than traditional shape indices. This is because the 444 new indices are derived based on the oriented bounding rectangles, in which just one of 445 the two patch parameters (patch area or patch perimeter) might be utilized. In traditional 446 shape indices, however, both of the two parameters are incorporated simultaneously. 447 This is why a high TD separability value for the moderately and severely saline soil 448 classes was obtained by traditional indices (Table 6). No single measurement or index 449 of shape can unambiguously differentiate all shapes (Forman 1995, Saura and Carballal 450 2004, Zhang et al. 2006). Combination of novel and traditional shape indices might be 451 necessary for some complex landscape analysis. In fact, the identification of the 452 severely saline soil patches combined both PPMBP and patch size.

\section{$453 \quad 5.5$ Future research}

454 The combination of minimum width bounding (MWB) box and moment bounding 455 (MB) box, offers a flexible approach for patch structural analysis. ODI, for example, 456 may be further divided into two categories: (1) $\mathrm{ODI}<\delta$, the patch is symmetrical; and 
(2) ODI $>\delta$, the patch is asymmetrical. For the case of $\mathrm{ODI}<\delta$, two situations can be

458 further divided: patch symmetry around the major axis of the MB box, and patch 459 symmetry around the minor axis of the MB box. In fact, some patches of the moderately 460 saline soil developed asymmetrically around the long sides of a patch (e.g. Figure 3(a)) 461 often belonging to the latter; whereas most of the slightly saline soil patches belong to 462 the former (e.g. Figure 3(b)). At the same time, a positive direction index can be 463 assigned to the patch once the ratio between the distance of a patch centroid to the 464 MWB box's centroid and half of the MWB box length surpasses a user defined 465 threshold (e.g., the positive direction of the patch shown by Figure 3(b) is from left to 466 right). For the case of ODI $>\delta$, two situations, namely left-handed rotation and right467 handed rotation may further be deduced according to the relations between the two 468 major axes. All these cases illustrate that patch heterogeneity can appear at the two ends 469 of the major or minor axis, or around one of the axes. Moreover, new shape metrics for 470 purely geometric representation might be deduced. For example, indices of "L-shape", 471 "T-shape" "cross-shape (+)", etc. might be designed for building object-based remote 472 sensing image segmentation. At the same time, as explained above, the proposed indices 473 have great potential utility in a wide range of applications, including landscape ecology. 474 Future research should be undertaken both to investigate the applicability and utility of 475 the proposed techniques in these fields, as well as to develop them further.

\section{$476 \quad 6$ Conclusion}

477 Two minimal bounding rectangles (i.e. minimal width bounding (MWB) box and 478 moment bounding (MB) box), suitable for anisotropic landscape analysis, were 479 introduced in this research. Moreover, four new shape metrics, namely MBLW (the 480 length-to-width ratio of MB box), PAMBA (area ratio between patch and MB box), PPMBP (perimeter ratio between patch and MB box) and ODI (orientation difference 
482 index between MB and MWB boxes), were introduced to quantify multiple aspects of

483 landscape pattern including patch elongation, patch compactness, patch roughness and

484 patch symmetry. These boxes and indices allow quantification of patch directionality

485 and shape complexity simultaneously, which is especially suitable for anisotropic

486 landscape pattern analysis. The experiment with real landscape data consisting of three

487 saline soil classes demonstrated that the proposed indices measure multiple geometric

488 dimensions of an anisotropic landscape, and led to a more accurate and robust

489 classification of soil type than traditional shape indices.

\section{Acknowledgements}

491 This research was supported by the European Union Erasmus Mundus Scholarship

492 [grant number 2011-0155] and co-funded by the Lancaster University Research Fund.

493 The authors are grateful to Professor Shanwen Qiu at Northeast Institute of Geography

494 and Agro-ecology, Chinese Academy of Sciences, for his expertise on saline soil rule

495 set development. The authors would also like to thank the two anonymous referees for 496 their constructive comments on this manuscript.

\section{References}

498 Baker, W.L. and Cai, Y., 1992. The r.le programs for multiscale analysis of landscape structure

499 using the GRASS geographical information system. Landscape Ecology, 7 (4), 291-302.

500 Bolliger, J., Wagner, H.H., and Turner, M.G., 2007. Identifying and Quantifying Landscape

$501 \quad$ Patterns in Space and Time. A Changing World. Springer Netherlands.

502 Chaudhuri, D. and Samal, A., 2007. A simple method for fitting of bounding rectangle to closed 503 regions. Pattern Recognition, 40 (7), 1981-1989.

504 Chi, C.-M. and Wang, Z.-C., 2010. Characterizing Salt-Affected Soils of Songnen Plain Using

$505 \quad$ Saturated Paste and 1:5 Soil-to-Water Extraction Methods. Arid Land Research and

$506 \quad$ Management, 24 (1), 1-11.

507 Cissel, J.H., Swanson, F.J., and Weisberg, P.J., 1999. Landscape management using historical 
509 Corry, R.C. and Nassauer, J.I., 2005. Limitations of using landscape pattern indices to evaluate 510 the ecological consequences of alternative plans and designs. Landscape and Urban

$511 \quad$ Planning, 72 (4), 265-280.

512 Feder, J., 1988. Fractals. New York, NY, USA: Plenium Press.

513 Forman, R.T.T., 1995. Some general principles of landscape and regional ecology. Landscape Ecology, 10 (3), 133-142.

515 Forman, R.T.T. and Godron, M., 1986. Landscape Ecology. New York, NY, USA: John Wiley 516 and Sons, Inc.

517 Fu, B. and Chen, L., 2000. Agricultural landscape spatial pattern analysis in the semi-arid hill

518 area of the Loess Plateau, China. Journal of Arid Environments, 44 (3), 291-303.

519 Gardner, R.H. and O'Neill, R. V, 1991. Pattern, process, and predictability: the use of neutral models for landscape analysis. Quantitative methods in landscape ecology. New York, NY, USA: Springer-Verlag.

Garrabou, J., Riera, J., and Zabala, M., 1998. Landscape pattern indices applied to mediterranean subtidal rocky benthic communities. Landscape Ecology, 13 (4), 225-247.

Gertsev, V.I., 2004. Classification of mathematical models in ecology. Ecological Modelling, 178 (3-4), 329-334.

Grimm, V., Revilla, E., Berger, U., Jeltsch, F., Mooij, W.M., Railsback, S.F., Thulke, H.-H., Weiner, J., Wiegand, T., and DeAngelis, D.L., 2005. Pattern-oriented modeling of agentbased complex systems: lessons from ecology. Science, 310 (5750), 987-991.

Gustafson, E.J., 1998. Quantifying Landscape Spatial Pattern: What Is the State of the Art? Ecosystems, 1 (2), 143-156.

Gustafson, E.J. and Parker, G.R., 1992. Relationships between landcover proportion and indices of landscape spatial pattern. Landscape Ecology, 7 (2), 101-110.

Helfenstein, J., Bauer, L., Clalüna, A., Bolliger, J., and Kienast, F., 2014. Landscape ecology meets landscape science. Landscape Ecology, 29 (7), 1109-1113. based analysis to examine the success of forest policy implementation in Southwest China. Landscape Ecology, 30 (6), 1111-1127.

538 Huang, Y., Yang, G., Gu, J., Wang, P., Huang, Q., Feng, Z., and Feng, L., 2013. Marine incursion events in the Late Cretaceous Songliao Basin: Constraints from sulfur 

161.

542 Hulshoff, R.M., 1995. Landscape indices describing a Dutch landscape. Landscape Ecology, 10 (2), 101-111.

Jiao, L., Liu, Y., and Li, H., 2012. Characterizing land-use classes in remote sensing imagery by shape metrics. ISPRS Journal of Photogrammetry and Remote Sensing, 72, 46-55.

Kaplan, D.A., Paudel, R., Cohen, M.J., and Jawitz, J.W., 2012. Orientation matters: Patch anisotropy controls discharge competence and hydroperiod in a patterned peatland. Geophysical Research Letters, 39 (17), 1-7.

Krummel, J.R., Gardner, R.H., Sugihara, G., O’Neill, R. V., and Coleman, P.R., 1987. Landscape Patterns in a Disturbed Environment. Oikos, 48 (3), 321-324.

Kupfer, J.A., 2012. Landscape ecology and biogeography: Rethinking landscape metrics in a post-FRAGSTATS landscape. Progress in Physical Geography, 36 (3), 400-420.

Lausch, A., Blaschke, T., Haase, D., Herzog, F., Syrbe, R.-U., Tischendorf, L., and Walz, U., 2015. Understanding and quantifying landscape structure - A review on relevant process characteristics, data models and landscape metrics. Ecological Modelling, 295, 31-41.

Leduc, A., Prairie, Y.T., and Bergeron, Y., 1994. Fractal dimension estimates of a fragmented landscape: sources of variability. Landscape Ecology, 9 (4), 279-286.

Legendre, P. and Fortin, M.J., 1989. Spatial pattern and ecological analysis. Vegetatio, 80 (2), 107-138.

Lewis, H.G., Cote, S., and Tatnall, A.R.L., 1997. Determination of spatial and temporal characteristics as an aid to neural network cloud classification. International Journal of Remote Sensing, 18 (4), 899-915.

Li, B.L., 2000. Fractal geometry applications in description and analysis of patch patterns and patch dynamics. Ecological Modelling, 132 (1-2), 33-50.

Li, Y., Li, Y., Qureshi, S., Kappas, M., and Hubacek, K., 2015. On the relationship between landscape ecological patterns and water quality across gradient zones of rapid urbanization in coastal China. Ecological Modelling, 318, 100-108.

Markofsky, S. and Bevan, A., 2012. Directional analysis of surface artefact distributions: A case study from the Murghab Delta, Turkmenistan. Journal of Archaeological Science, 39 (2), 428-439.

McGarigal, K. and McComb, W.C., 1995. Relationships between landscape structure and 
breeding birds on the Oregon Coast Range. Ecological Monographs, 65 (3), 235-260.

573 McGarigal, K., Tagil, S., and Cushman, S.A., 2009. Surface metrics: An alternative to patch metrics for the quantification of landscape structure. Landscape Ecology, 24 (3), 433-450.

O’Neill, R. V., Krummel, J.R., Gardner, R.H., Sugihara, G., Jackson, B., DeAngelis, D.L., Milne, B.T., Turner, M.G., Zygmunt, B., Christensen, S.W., Dale, V.H., and Graham, R.L., 1988. Indices of landscape pattern. Landscape Ecology, 1 (3), 153-162.

Qiu, S., Wang, X., Zhang, S., Lian, Y., Zhang, Z., and Zhu, J., 2012. The evolution of the large paleolake in Songliao Plain and its formation. Quaternary Sciences, 32 (5), $1011-1021$ (in Chinese).

Reddy, C.S., Sreelekshmi, S., Jha, C.S., and Dadhwal, V.K., 2013. National assessment of forest fragmentation in India: Landscape indices as measures of the effects of fragmentation and forest cover change. Ecological Engineering, 60, 453-464.

Remmel, T.K. and Csillag, F., 2003. When are two landscape pattern indices significantly different? Journal of Geographical Systems, 5 (4), 331-351.

Remmel, T.K. and Fortin, M.J., 2013. Categorical, class-focused map patterns: Characterization and comparison. Landscape Ecology, 28 (8), 1587-1599.

Riitters, K.H., O’Neill, R. V., Hunsaker, C.T., Wickham, J.D., Yankee, D.H., Timmins, S.P., Jones, K.B., and Jackson, B.L., 1995. A factor analysis of landscape pattern and structure metrics. Landscape Ecology, 10 (1), 23-39.

Rossi, R.E., Mulla, D.J., Journel, A.G., and Franz, E.H., 1992. Geostatistical tools for modeling and interpreting ecological spatial dependence. Ecological Monographs, 62 (2), 277-314.

Saura, S. and Carballal, P., 2004. Discrimination of native and exotic forest patterns through shape irregularity indices: An analysis in the landscapes of Galicia, Spain. Landscape Ecology, 19 (6), 647-662.

Schröder, B. and Seppelt, R., 2006. Analysis of pattern-process interactions based on landscape models-Overview, general concepts, and methodological issues. Ecological Modelling, 199 (4), 505-516.

Seto, K.C. and Fragkias, M., 2005. Quantifying spatiotemporal patterns of urban land-use

601 change in four cities of China with time series landscape metrics. Landscape Ecology, 20

602 (7), 871-888.

Stigler, S.M., 1981. Gauss and the Invention of Least Squares. The Annals of Statistics, 9 (3), 
Syrbe, R.U. and Walz, U., 2012. Spatial indicators for the assessment of ecosystem services: Providing, benefiting and connecting areas and landscape metrics. Ecological Indicators, $21,80-88$.

Timoshenko, S. and Gere, J.M., 1972. Mechanics of Materials. New York, NY, USA: Van Nostrand Reinhold Co.

Tischendorf, L., 2001. Can landscape indices predict ecological processes consistently? Landscape Ecology, 16 (3), 235-254.

Toussaint, G., 1983. Solving geometric problems with the rotating calipers. In: IEEE MELECON83. 1-8.

Turner, M.G., 1990. Spatial and temporal analysis of landscape patterns. Landscape Ecology, 4 (1), 21-30.

Turner, M.G., 2005. Landscape Ecology: What Is the State of the Science? Annual Review of Ecology, Evolution, and Systematics, 36, 319-344.

Uuemaa, E., Mander, Ü., and Marja, R., 2013. Trends in the use of landscape spatial metrics as landscape indicators: A review. Ecological Indicators, 28, 100-106.

Vidal Vázquez, E., Vivas Miranda, J.G., and Paz González, A., 2005. Characterizing anisotropy and heterogeneity of soil surface microtopography using fractal models. Ecological Modelling, 182 (3-4), 337-353.

Vogt, P., Riitters, K.H., Estreguil, C., Kozak, J., Wade, T.G., and Wickham, J.D., 2007. Mapping spatial patterns with morphological image processing. Landscape Ecology, 22 (2), 171-177.

Wang, L., Seki, K., Miyazaki, T., and Ishihama, Y., 2009. The causes of soil alkalinization in the Songnen Plain of Northeast China. Paddy and Water Environment, 7 (3), 259-270.

Wu, J., 2013. Key concepts and research topics in landscape ecology revisited: 30 years after the Allerton Park workshop. Landscape Ecology, 28 (1), 1-11.

Wu, J., Jelinski, D.E., Luck, M., and Tueller, P.T., 2000. Multiscale Analysis of Landscape Heterogeneity: Scale Variance and Pattern Metrics. Annals of GIS, 6 (1), 6-19.

Yuan, J., Cohen, M.J., Kaplan, D.A., Acharya, S., Larsen, L.G., and Nungesser, M.K., 2015. Linking metrics of landscape pattern to hydrological process in a lotic wetland. Landscape Ecology, 30 (10), 1893-1912.

Zaslavsky, D. and Rogowski, A.S., 1969. Hydrologic and Morphologic Implications of Anisotropy and Infiltration in Soil Profile Development. Soil Science Society of America 
Journal, 33 (4), 594-599.

637 Zhang, S., Zhang, J., Li, F., and Cropp, R., 2006. Vector analysis theory on landscape pattern 638 (VATLP). Ecological Modelling, 193 (3-4), 492-502.

639 
641 Table 1 Detailed description of novel shape metrics.

\begin{tabular}{lll}
\hline Indices (Acronym) & Formula & Description \\
\hline & & Description of vector patch elongatedness \\
patch length-to- & According to the ratio between length (m) \\
width ratio & L/W & and width (m) of MB: The MBLW $\geqq 1$. \\
& & The lager the value, the more elongated the \\
& & shape.
\end{tabular}

\section{Description of vector patch compactness}

Area ratio between

patch and MB box

(PAMBA)
According to the ratio between patch area

PA/MBA (ha) and MB area (ha): The larger the value, the larger the filling degree and the more compact the shape.

\section{Description of vector patch roughness}

Perimeter ratio

between patch and

MB box (PPMBP)

PP/MBP

According to the ratio between patch

perimeter $(\mathrm{m})$ and $\mathrm{MB}$ perimeter $(\mathrm{m})$ : The

larger the value, the rougher the patch's

edge.

\section{Description of vector patch symmetry}

Orientation

If $\mathrm{ODI}<\delta$ ( $\delta$ is user defined threshold),

difference (0-180)

$\left|\theta_{M B}-\theta_{M W B}\right|$

between MB and

MWB (ODI) the patch is symmetric; the smaller (the larger respectively) the value, the more symmetrical (asymmetrical respectively) the shape. 
643 Table 2 Rule sets based on novel shape indices for saline soil feature extraction.

\begin{tabular}{ccc}
\hline Class & Shape indices & Rules \\
\hline slightly & ODI & $<=4.6$ \\
saline soil & MBLW & $>3$ \\
& PAMBA & $>0.34$ \\
\hline MBLW & $<2.8$ \\
saline soil & PAMBA & $(0.18-0.57)$ \\
& PPMBP & $<2.22$ \\
\hline severely & Patch area & $>=4,000.00$ (ha) \\
saline soil & PPMBP & $>3.4$
\end{tabular}

644

Note: Intersection set operations within rule sets

645 
646 Table 3 Area and number of patches of each saline soil class.

\begin{tabular}{cccccc}
\hline $\begin{array}{c}\text { Saline soil } \\
\text { class }\end{array}$ & $\begin{array}{c}\text { Patch } \\
\text { numbers }\end{array}$ & $\begin{array}{c}\text { Mean patch } \\
\text { area (ha) }\end{array}$ & $\begin{array}{c}\text { total area } \\
\text { (ha) }\end{array}$ & $\begin{array}{c}\text { Mean patch } \\
\text { perimeter }(\mathrm{m})\end{array}$ & $\begin{array}{c}\text { Total perimeter } \\
(\mathrm{m})\end{array}$ \\
\hline $\begin{array}{c}\text { slightly } \\
\text { saline soil }\end{array}$ & 45 & $3,417.18$ & $153,773.10$ & $83,983.36$ & $3,779,251.35$ \\
\hline moderately & 127 & 521.67 & $66,251.92$ & $20,805.78$ & $2,642,333.99$ \\
saline soil & $126,026.68$ & $130,133.42$ & $664,982.95$ & $3,324,914.95$ \\
\hline $\begin{array}{l}\text { severely } \\
\text { saline soil }\end{array}$ & 5 & 26,05 & & \\
\hline
\end{tabular}

647 
648 Table 4 Feature separability of novel shape indices corresponding to the rule sets.

\begin{tabular}{|c|c|c|c|c|}
\hline Shape indices & Saline soil class & $\begin{array}{c}\text { slightly } \\
\text { saline soil }\end{array}$ & $\begin{array}{l}\text { Moderately } \\
\text { saline soil }\end{array}$ & $\begin{array}{l}\text { Severely } \\
\text { saline soil }\end{array}$ \\
\hline \multirow{2}{*}{ ODI, MBLW } & Slightly saline soil & - & 1.7593 & 1.9857 \\
\hline & Moderately saline soil & 1.9361 & $\longrightarrow$ & 1. 3408 \\
\hline and PAMBA & Severely saline soil & 2.0000 & 1.4843 & - \\
\hline MBLW, & Slightly saline soil & - & 1.9316 & 1.7562 \\
\hline PAMBA and & Moderately saline soil & 1.9685 & - & 1. 9408 \\
\hline PPMBP & Severely saline soil & 1.8741 & 1.9843 & - \\
\hline \multirow{3}{*}{$\begin{array}{c}\text { Patch area } \\
\text { and PPMBP }\end{array}$} & Slightly saline soil & - & 0.8335 & 1.9441 \\
\hline & Moderately saline soil & 1.3408 & - & 1.9343 \\
\hline & Severely saline soil & 1.9961 & 2.0000 & - \\
\hline
\end{tabular}

649 
650 Table 5 Detailed description of traditional shape metrics.

\begin{tabular}{|c|c|c|}
\hline Shape index & Formula & Description \\
\hline $\begin{array}{l}\text { Mean perimeter- } \\
\text { area ratio } \\
(M P A R)\end{array}$ & $M P A R=\frac{p}{a}$ & $\begin{array}{l}\text { The ratio between patch } \\
\text { perimeter }(\mathrm{m}) \text { and area }(\mathrm{ha})\end{array}$ \\
\hline $\begin{array}{l}\text { fractal dimension } \\
\qquad(F D)\end{array}$ & $F D=\frac{2 \ln (p)-\ln (k)}{\ln a}$ & Here $k=1$. \\
\hline Shape index $(S I)$ & $S I=\frac{p}{2 \sqrt{\pi} \sqrt{a}}$ & $\begin{array}{l}S I \text { attains its minimum }(S I= \\
\text { 1) for circles and increases } \\
\text { (with no upper limit) for more } \\
\text { complex or elongated shapes }\end{array}$ \\
\hline
\end{tabular}

651 Note: $p$ and $a$ are, respectively, the perimeter and area of the patch

652 
653 Table 6 The TD separability and JM distance (italic) of the three traditional shape 654 indices.

\begin{tabular}{cccc}
\hline Saline soil class & $\begin{array}{c}\text { Slightly } \\
\text { saline soil }\end{array}$ & Moderately & Severely \\
& & saline soil & saline soil \\
\hline Slightly saline soil & - & 1.1129 & 1.7397 \\
Moderately saline soil & 1.2962 & - & 1.4886 \\
Severely saline soil & 1.8212 & 1.617 & - \\
\hline
\end{tabular}


656 Figure captions

657 Figure 1. A patch $(\mathrm{S})$ with centroid $\mathrm{C}(\bar{x}, \bar{y}), d A$ is the differential area of point $(x, y)$, 658 oxy is the geographic coordinate system.

659 Figure 2. A polygon with its MWB box (in blue) and MB (in red) box.

660 Figure 3. A polygon with its MWB box and MB box completely coincident. $\mathrm{C}$ is the 661 centroid of the polygon, and $\mathrm{AB}$ and EF are the major and minor axes of the MWB box, 662 respectively, and $\mathrm{MN}$ and $\mathrm{PQ}$ are the major and minor axes of the $\mathrm{MB}$ box, 663 respectively. (a) the centroid lying on the minor axis of MWB; (b) the centroid lying on 664 the major axis of MWB.

665 Figure 4. The Geographic location of study area.

666 Figure 5. Part of study area showing (a) slightly saline soil patches with their MWB 667 and MB boxes, (b) moderately saline soil patches with their MWB and MB boxes and 668 (c) severely saline soil patches with their MWB and MB boxes.

669 Figure 6. Different saline soil classes identified by the rule sets developed by the 670 proposed novel indices.

671 Figure 7. A patch example with MB box (in red), MWB box (in blue) and MAB box 672 (in green).

673

674 


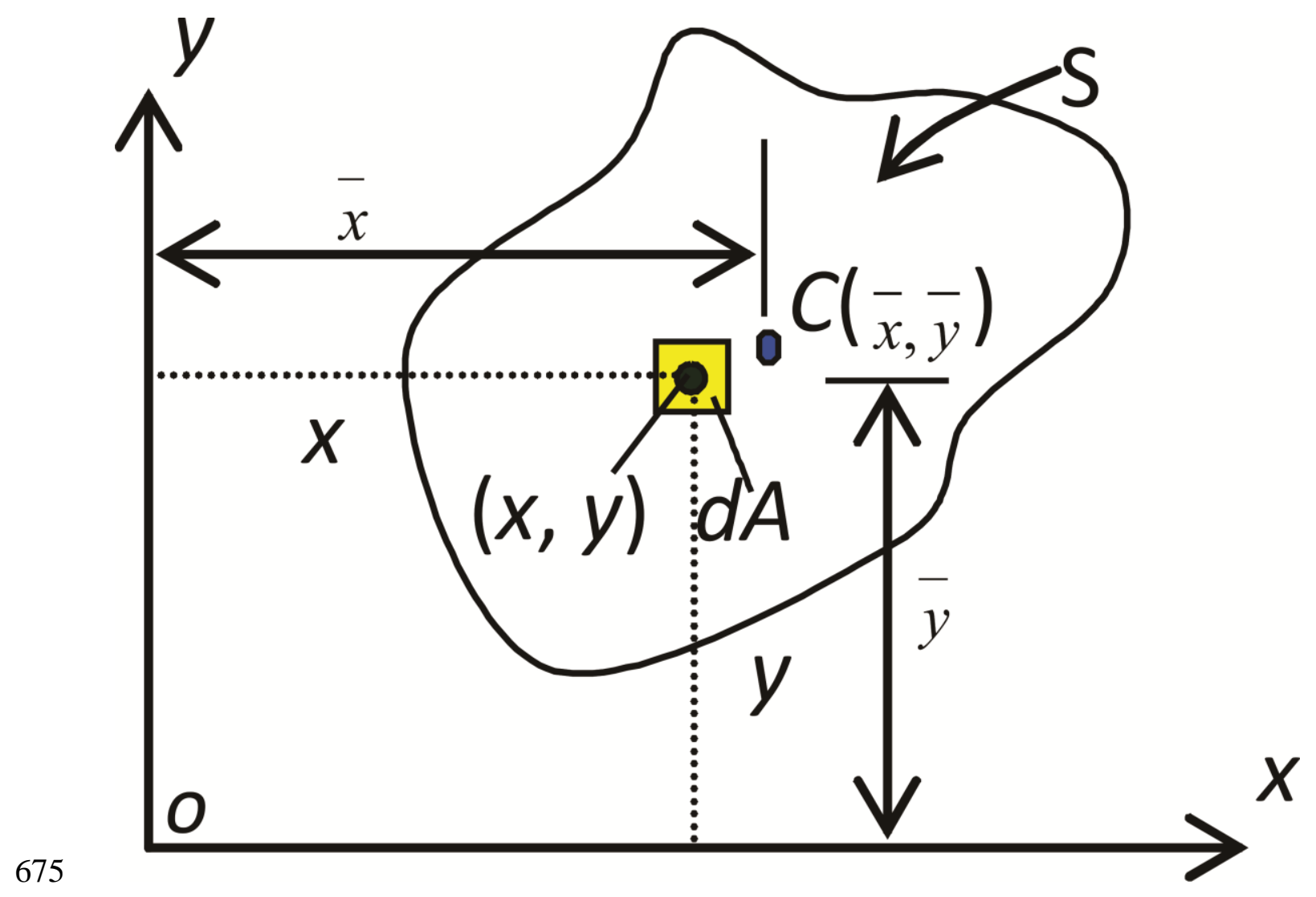

676 


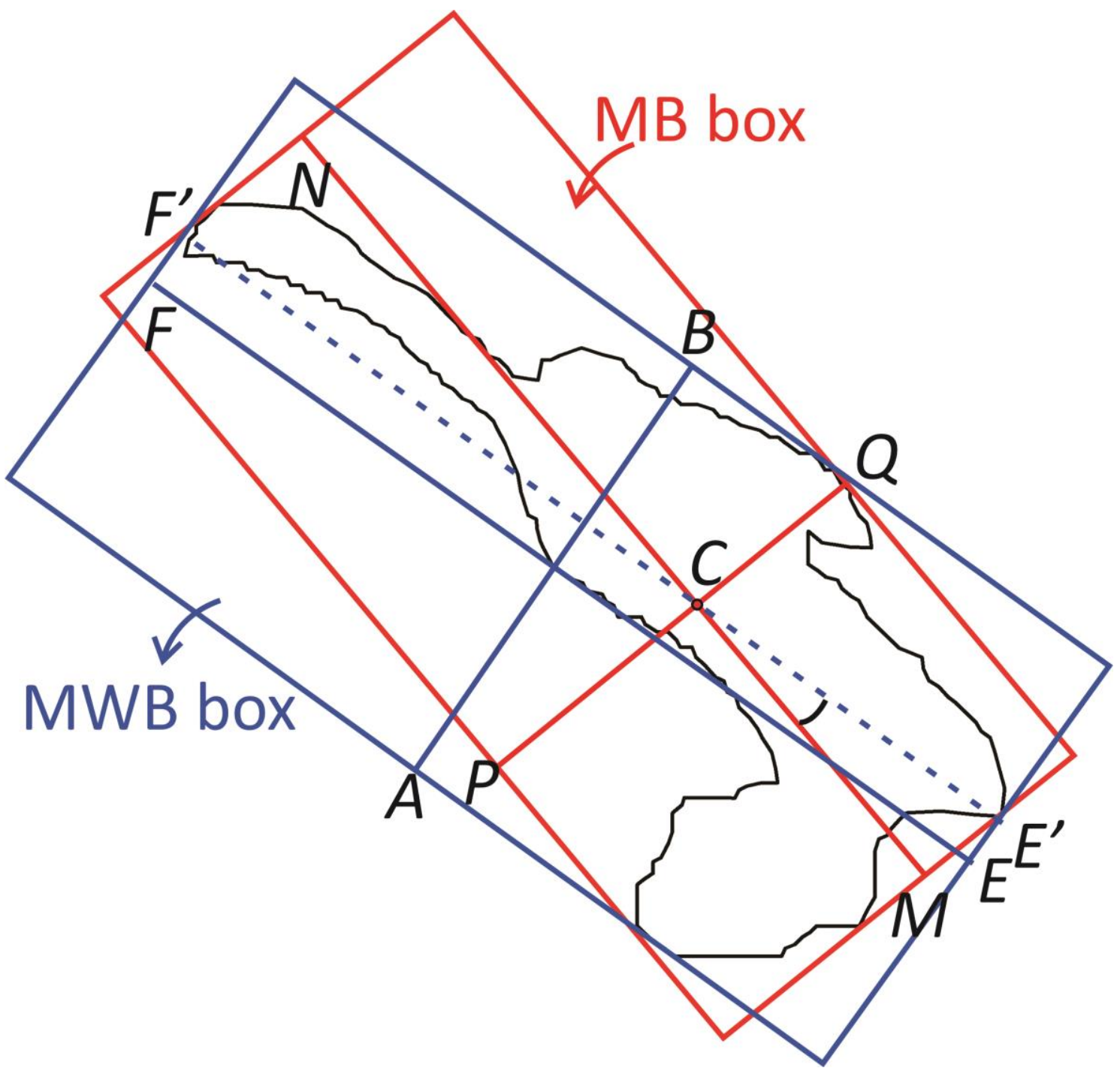

678 


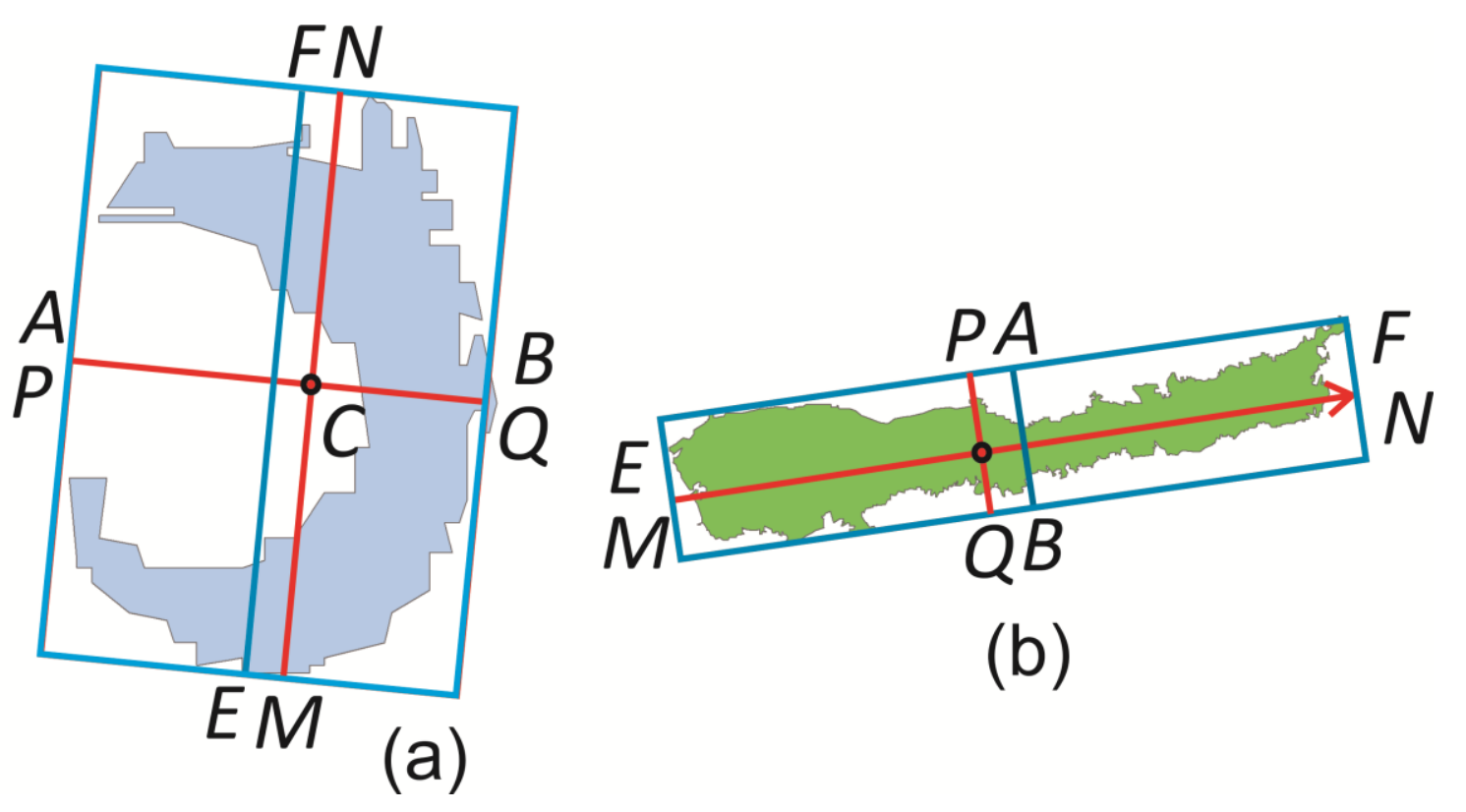

680 


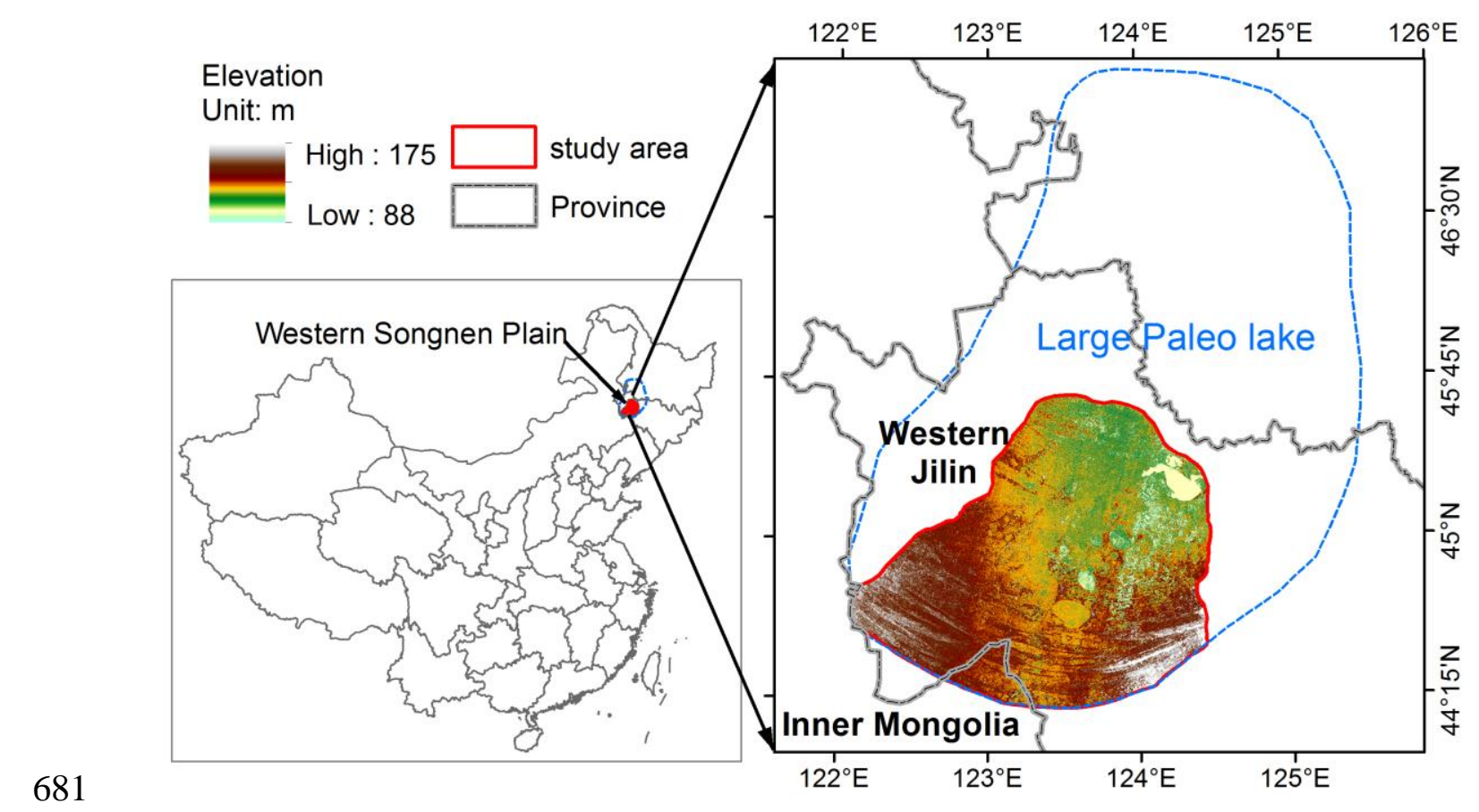

682 


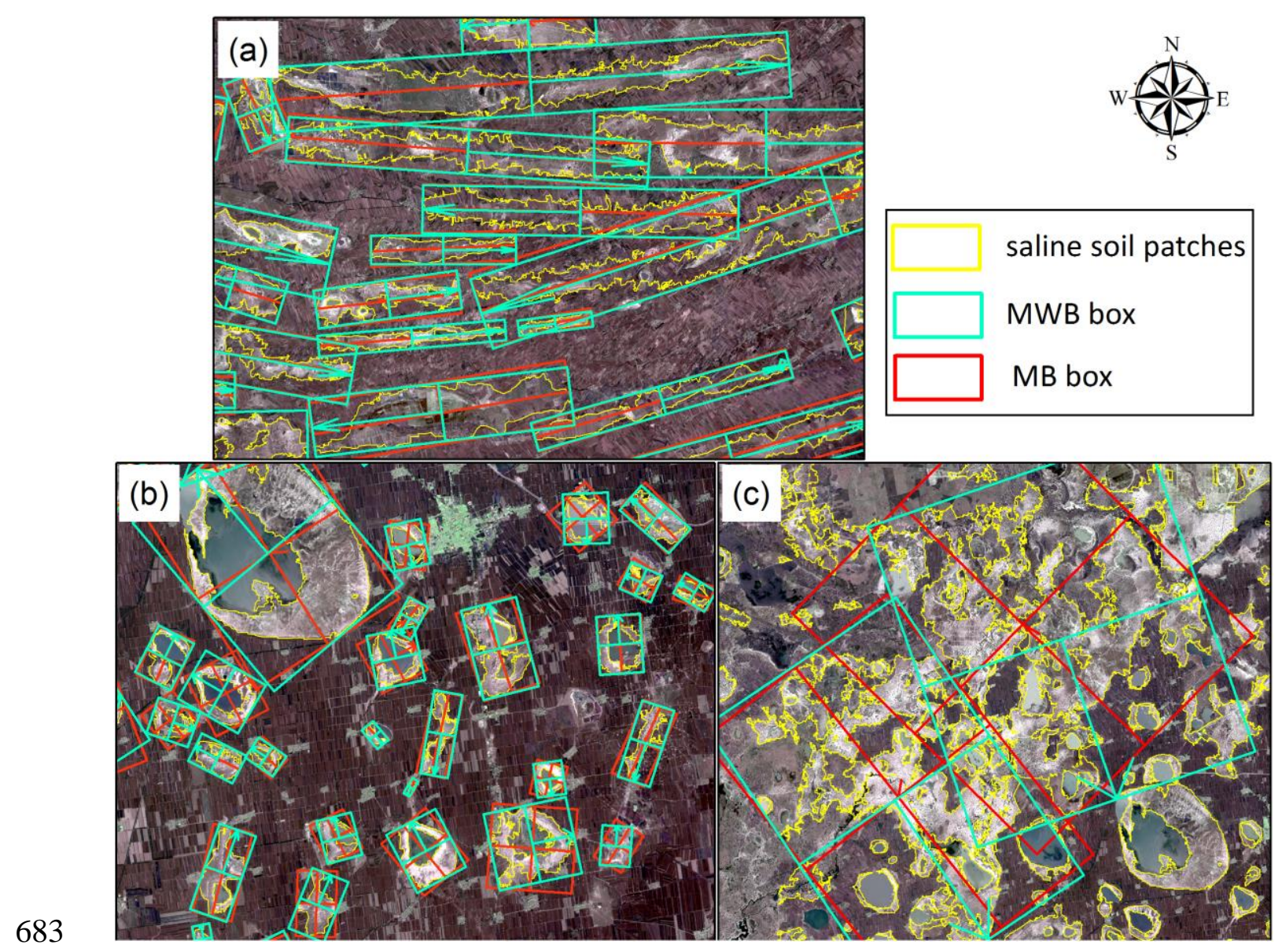

684 


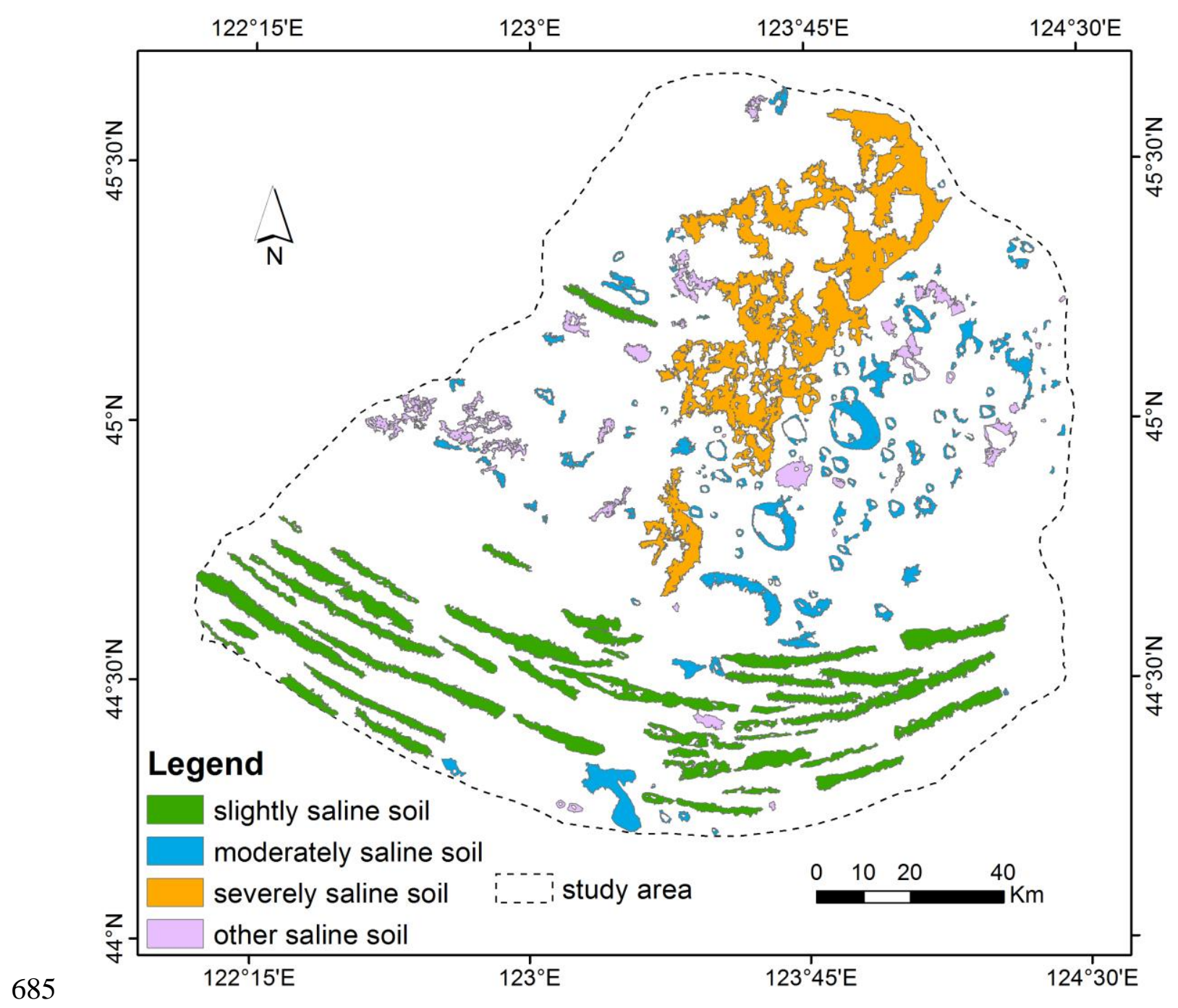

686 


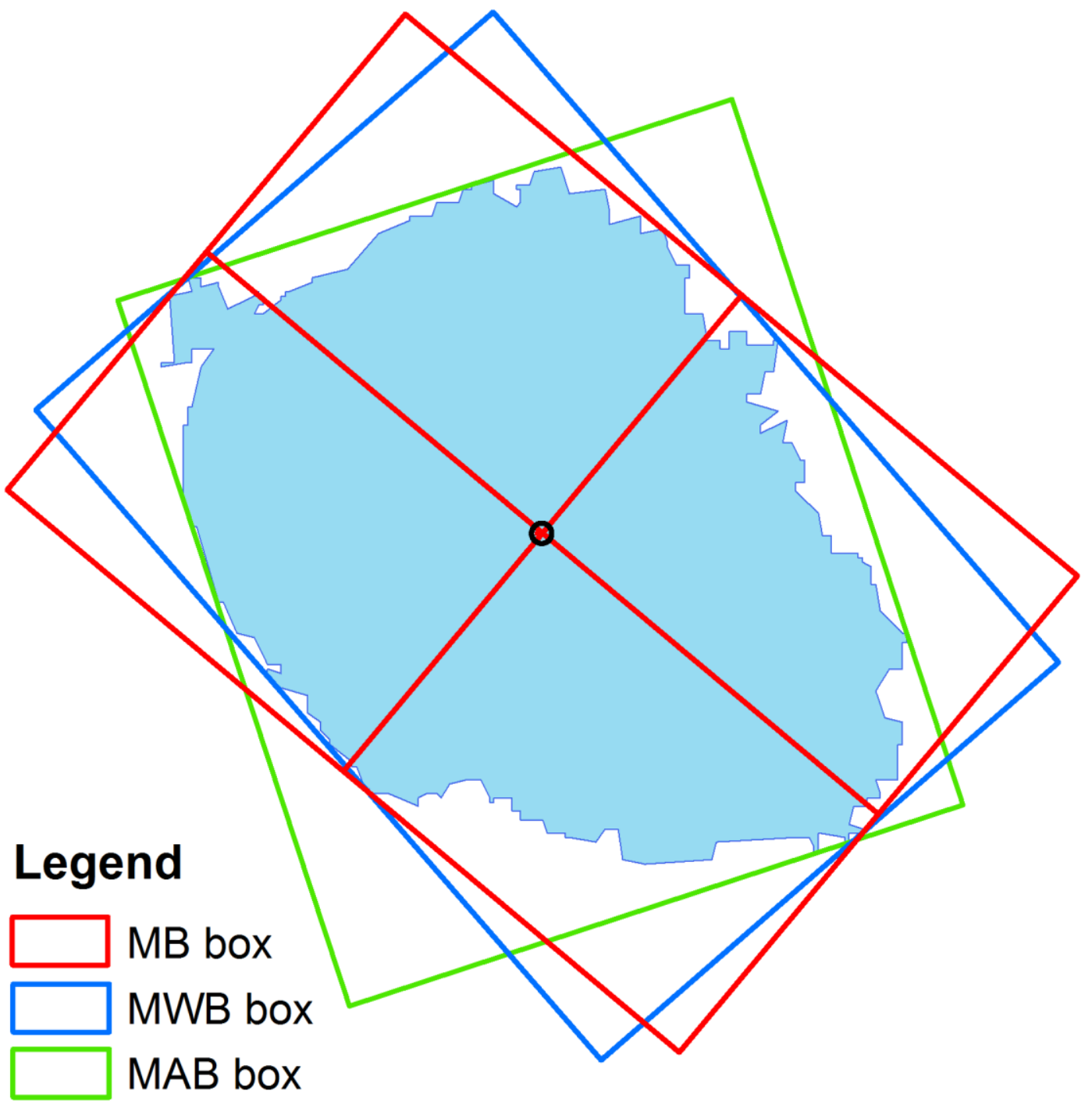

